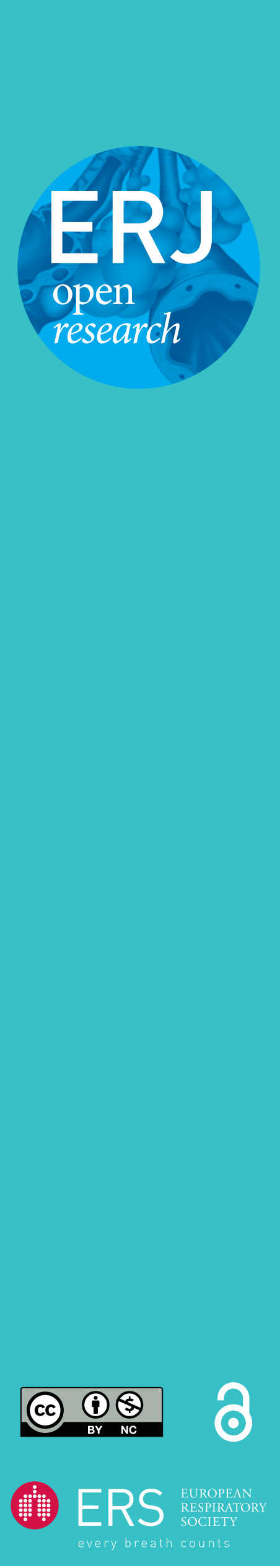

\title{
Summary of the international clinical guidelines for the management of hospital-acquired and ventilator-acquired pneumonia
}

\author{
Antoni Torres ${ }^{1}$, Michael S. Niederman ${ }^{2}$, Jean Chastre ${ }^{3}$, Santiago Ewig ${ }^{4}$, \\ Patricia Fernandez-Vandellos ${ }^{5}$, Hakan Hanberger ${ }^{6}$, Marin Kollef ${ }^{7}$, \\ Gianluigi Li Bassi ${ }^{1}$, Carlos M. Luna ${ }^{8}$, Ignacio Martin-Loeches ${ }^{9}$, \\ J. Artur Paiva ${ }^{10,11}$, Robert C. Read ${ }^{12}$, David Rigau ${ }^{13}$, Jean François Timsit ${ }^{14}$, \\ Tobias Welte ${ }^{15,16}$ and Richard Wunderink ${ }^{17}$
}

Affiliations: ${ }^{1}$ Dept of Pulmonology, Hospital Clínic de Barcelona, Universitat de Barcelona, IDIBAPS, CIBERES Barcelona, Barcelona, Spain. ${ }^{2}$ Division of Pulmonary and Critical Care Medicine, Weill Cornell Medicine, New York, NY, USA. ${ }^{3}$ Réanimation Médicale, Groupe Hospitalier Pitié-Salpêtrière, Paris, France. ${ }^{4}$ Thoraxzentrum Ruhrgebiet, Kliniken für Pneumologie und Infektiologie, Herne und Bochum, Germany. ${ }^{5}$ IDIBAPS, CIBER de Enfermedades Respiratorias (CIBERES), Barcelona, Spain. ${ }^{6}$ Dept of Clinical and Experimental Medicine, Faculty of Medicine and Health Sciences, Linköping University, Linköping, Sweden. ${ }^{7}$ Pulmonary and Critical Care Division, Washington University School of Medicine, St Louis, MO, USA. ${ }^{8}$ Dept of Medicine, Pulmonary Diseases Division, Hospital de Clínicas "José de San Martin", Universidad de Buenos Aires, Buenos Aires, Argentina. ${ }^{9}$ Dept of Clinical Medicine, Wellcome Trust - HRB Clinical Research Facility, St. James's Hospital, Trinity College, Dublin, Ireland. CIBER de Enfermedades Respiratorias (CIBERES). ${ }^{10}$ Emergency and Intensive Care Dept, Centro Hospitalar São João EPE, Porto, Portugal. ${ }^{11}$ Dept of Medicine, University of Porto Medical School, Porto, Portugal. ${ }^{12}$ NIHR Southampton Biomedical Research Centre, University Hospital Southampton, Southampton, UK. ${ }^{13}$ Iberoamerican Cochrane Center, Barcelona, Spain. ${ }^{14}$ IAME, INSERM UMR 1137, Medical and Infectious Diseases Intensive Care Unit, Paris Diderot University and Bichat Hospital, Paris, France. ${ }^{15}$ CAPNETZ STIFTUNG, Hannover, Germany. ${ }^{16}$ Dept of Respiratory Medicine, Medizinische Hoschschule Hannover and German Center of Lung Research (DZL), Germany. ${ }^{17}$ Division of Pulmonary and Critical Care Medicine, Northwestern University Feinberg School of Medicine, Chicago, IL, USA.

Correspondence: Antoni Torres, Servei de Pneumologia, Hospital Clinic de Barcelona, Villarroel 170, 08036 Barcelona, Spain. E-mail: atorresđub.edu

@ERSpublications

A summary of the evidence and recommendations made in the ERS/ESICM/ESCMID/ALAT guidelines for the management of hospital-acquired pneumonia and ventilator-associated pneumonia http://ow.ly/S3zA30iZfLa

Cite this article as: Torres A, Niederman MS, Chastre J et al. Summary of the international clinical guidelines for the management of hospital-acquired and ventilator-acquired pneumonia. ERJ Open Res 2018; 4: 00028-2018 [https://doi.org/10.1183/23120541.00028-2018].

The most recent European guidelines and task force reports on hospital-acquired pneumonia (HAP) and ventilator-associated pneumonia (VAP) were published almost 10 years ago. Since then, further randomised clinical trials of HAP and VAP have been conducted and new information has become available. Studies of epidemiology, diagnosis, empirical treatment, response to treatment, new antibiotics or new forms of antibiotic administration, and disease prevention have changed old paradigms. In addition, important differences between approaches in Europe and the USA have become apparent. The European Respiratory Society (ERS) launched a project to develop new international guidelines for HAP and VAP.

Received: Feb 142018 | Accepted: Feb 222018

Copyright $\odot$ ERS 2018. This article is open access and distributed under the terms of the Creative Commons Attribution Non-Commercial Licence 4.0. 
Other European societies, including the European Society of Intensive Care Medicine (ESICM) and the European Society of Clinical Microbiology and Infectious Diseases (ESCMID), were invited to participate and appointed their representatives. The Latin American Society of Thoracic Diseases (ALAT) was also invited to participate. This manuscript summarises the evidence and recommendations of these international guidelines on HAP and VAP.

\section{Background}

Nosocomial pneumonia is a frequent infection that is classified into two groups [1]: HAP, which develops in hospitalised patients after $48 \mathrm{~h}$ of admission, and does not require (but may include) artificial ventilation at the time of diagnosis [2,3]; and VAP, which occurs in intensive care unit (ICU) patients who have received mechanical ventilation for at least $48 \mathrm{~h}[2,3]$.

HAP is the second most common hospital infection and has the highest crude mortality, while VAP is the most frequent cause of nosocomial infection in the ICU $[2,3]$. Both types of pneumonia lengthen hospital stay and consume considerable health resources $[2,3]$.

Recently, the ERS, ESICM, ESCMID and ALAT published clinical guidelines on the therapeutic and management strategies for adult patients with HAP and VAP, designed to guide clinical decisions made not only by pulmonologists and intensivists but by all health professionals who treat these patients [2]. These clinical guidelines are evidence-based (Grading of Recommendations Assessment, Development and Evaluation) and follow the PICO (population-intervention-comparison-outcome) model.

Each section in this article refers to a specific PICO question addressed in the guidelines.

\section{Obtaining samples in intubated patients}

To identify the causative agent of nosocomial pneumonia, the recommendation is to perform a qualitative or quantitative (preferred) analysis of the respiratory secretions. In both cases, the quality of the respiratory samples should be assessed [3].

Both invasive techniques (fibre-optic bronchoscopy or mini-bronchoalveolar lavage) and noninvasive techniques can be used to obtain samples of respiratory secretions for quantitative or qualitative analysis. Examples of both types of technique listed in table 1.

Quantitative analyses use the bacteriological growth count to assist differentiation between infection (when there are high concentrations of a certain organism) and colonisation (when the concentrations are low); qualitative analyses focus on the presence or absence of pathogens [4].

Qualitative cultures of tracheal aspirates are simple and noninvasive, but the main methodological limitation is that samples are often contaminated by the flora colonising the upper tracts and are therefore less specific [5].

Invasive techniques also present several disadvantages: the need for qualified personnel to perform these procedures, potential risks for the patient, and the associated costs [2, 3]. Techniques such as bronchoalveolar lavage and the application of quantitative cultures achieve reliable identification of the causative agents of the infection, especially in patients at high risk, such as those undergoing mechanical ventilation [5], and can thus guide antibiotic treatment [2].

No randomised controlled clinical studies have compared quantitative and qualitative cultures of the same sample [2]. However, a Cochrane review from 2014 included five studies that compared invasive methods

\section{TABLE 1 Examples of invasive and noninvasive techniques for obtaining samples of respiratory} secretions

$\begin{array}{ll}\text { Technique } & \text { Example } \\ \text { Invasive } & \text { Fibre-optic bronchoscopy with protected specimen brush } \\ & \text { Fibre-optic bronchoscopy with alveolar lavage }{ }^{\#} \\ & \text { Lung biopsy and tissue culture } \\ \text { Noninvasive } & \text { Simple culture of endotracheal aspirate (qualitative) } \\ & \text { Quantitative culture of endotracheal aspirate } \\ & \text { Blind culture with protected/plugged telescopic catheter }{ }^{\#} \\ & \text { Blind protected specimen brush }{ }^{\#} \\ & \text { Blind alveolar lavage }\end{array}$


using quantitative cultures with noninvasive ones using qualitative cultures [4]. The authors concluded that there was no clear advantage between quantitative and qualitative cultures, or invasive and noninvasive techniques [4].

Noninvasive methods may overestimate the presence of bacteria in the initial examination of the samples, which can lead to excessive antibiotic use [2]. If the sample is obtained before changing the antibiotic treatment and yields a negative result, the antibiotic can be withdrawn since the possibility of no infection is high $[6,7]$. However, a negative result may also indicate that the infection has been controlled [2].

\section{Key points}

- The European guidelines indicate that it is preferable to obtain the distal quantitative samples before antibiotic treatment [2], since it is known that if samples are obtained within $48 \mathrm{~h}$ of the start of antibiotic treatment the result may be altered or emerge as negative $[2,5]$.

- In view of the general overuse of antibiotics, the reduction in their prescription through use of distal quantitative cultures may have a positive impact on the appearance of resistance and consequently on health expenditure, although no studies of cost effectiveness have been carried out to date [2].

\section{Treatment of early nosocomial pneumonia (before 5 days) and late nosocomial pneumonia (after 5 days)}

A distinction should be made between patients with risk factors for antibiotic resistance and patients without these risk factors, and also between early and late episodes.

In early VAP without risk factors, the most frequent causative pathogens are Streptococcus pneumoniae, Haemophilus influenzae and methicillin-sensitive Staphylococcus aureus. By contrast, in late VAP the causative agents are at high risk for antibiotic resistance: for example, Pseudomonas aeruginosa, Acinetobacter baumannii, methicillin-resistant S. aureus (MRSA) and other Gram-negative bacilli, depending on the predominant microorganism in each hospital and ICU [5]. Gram-negative bacilli are considered multidrug resistant (MDR) if they are resistant to three or more families of antibiotics to which they are normally sensitive, including $\beta$-lactams (penicillins and cephalosporins), carbapenems, aminoglycosides and quinolones [8]. The rate of resistant pathogens in local microbiological data is considered high when it exceeds $25 \%$ (figure 1) [2].

Patients with early nosocomial pneumonia who do not have risk factors for multidrug resistance, have a low mortality risk, and are treated in an ICU with a low rate of MDR pathogens, should be treated with antibiotics that cover community pathogens, while in patients with late nosocomial pneumonia or who present risk factors for multidrug resistance or a high risk of mortality, initial broad spectrum empirical treatment should be performed [3]. In the latter case, a combination of antibiotics should be prescribed in order to expand the spectrum and avoid resistance [3].

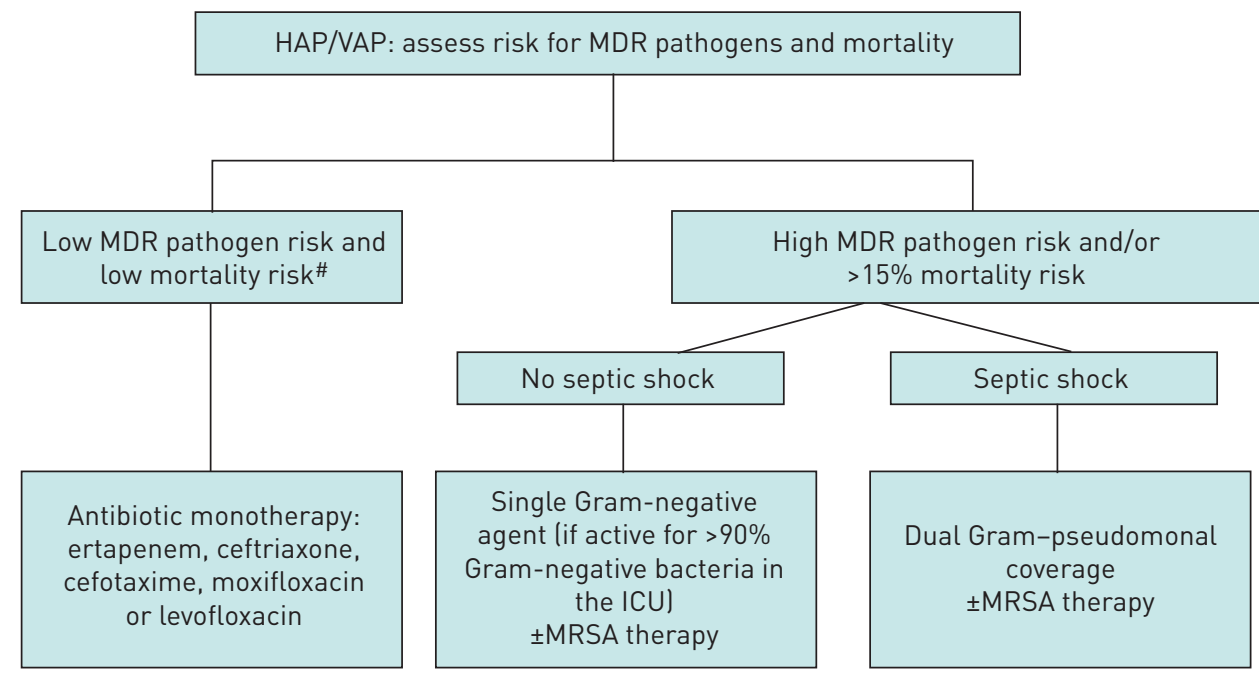

FIGURE 1 Empirical antibiotic treatment algorithm for hospital-acquired pneumonia (HAP)/ ventilator-associated pneumonia (VAP). MDR: multidrug-resistant; ICU: intensive care unit; MRSA: methicillin-resistant Staphylococcus aureus. \#: low risk for mortality is defined as a $\leqslant 15 \%$ chance of dying, a mortality rate that has been associated with better outcomes using monotherapy than combination therapy when treating serious infection [18]. Reproduced from [2]. 
It is necessary to define the nosocomial pneumonia patients who can receive narrow-spectrum empirical treatment and those who should receive broad-spectrum empirical treatment, in order to prevent the appearance of resistance and secondary effects related to antibiotics [2]. Patients susceptible to colonisation by MDR microorganisms usually present with more severe condition, are often immunocompromised and present risk factors, as is the case with many patients requiring mechanical ventilation [8].

The European guidelines defined low-risk patients as those who do not present with septic shock, do not have other risk factors for MDR pathogens and are not admitted to ICUs with a high rate of resistant pathogens (25\%) [2].

An important variable for identifying MDR pathogens is the time of pneumonia onset. Early HAP and VAP are defined by an occurrence within 4 days of hospital admission and are associated with a better prognosis, since they are more likely to be caused by microorganisms that are generally sensitive to antibiotics. Late-onset pneumonias develop after 5 days of hospitalisation and are more likely to be caused by MDR microorganisms, with higher morbidity and mortality [2,9]. Prior antimicrobial therapy or hospitalisation for longer than 2 days during the 3 months prior to the episode have also been identified as risk factors [2].

Previous studies found advanced age and previous use of antibiotics to be risk factors for MDR pathogens in ICU-acquired pneumonia $[2,10]$. Also, patients over 65 years of age are at a greater risk of infection with methicillin-resistant S. aureus [11], and acute renal failure is associated with an increased risk of community-acquired pneumonia due to P. aeruginosa, Enterobacteria producing extended spectrum $\beta$-lactamase (ESBL) and multidrug-resistance $[2,12]$.

Despite the lack of cost-effectiveness studies, the European guidelines consider that narrow-spectrum antibiotic treatment is associated with fewer direct costs, since the costs incurred by drug-related toxicity are lower and the emergence of multidrug resistance may also be reduced [2]. By contrast, administration of an inadequate narrow-spectrum antibiotic may entail higher costs due to the prolongation of mechanical ventilation and hospital stay and a greater final use of antibiotics [9].

Key points

- The selection of patients with early-onset nosocomial pneumonia to receive empirical treatment with narrow-spectrum antibiotics should be based on the individual risks, the severity of the clinical situation (shock), and the frequency and type of MDR pathogens detected in the ICU [2].

- Narrow-spectrum antibiotics can be used in patients with early onset of HAP or VAP, whenever the risk of resistance and mortality is considered to be low [2].

- In patients with early-onset HAP/VAP who develop septic shock, a broad-spectrum empirical treatment against $P$. aeruginosa and ESBL producing organisms is recommended [2]. In addition, MRSA should be covered if prevalent in the unit ( $>25 \%$ S. aureus in the unit).

- Multiple broad-spectrum antibiotics should not be used routinely, in order to prevent drug-related toxicities, such as renal failure due to the use of the nephrotoxic drugs aminoglycosides and vancomycin, but may be necessary in selected patients [2].

\section{One or two antibiotics for initial broad-spectrum empirical treatment}

The aims of combined antibiotic treatment are to obtain synergies between different groups of antibiotics, extending the spectrum of action against Gram-negative bacilli and avoiding the emergence of resistance $[3,8]$. However, combined treatments may also have disadvantages (at least potentially) such as the increased risk of toxicity, the higher cost of treatment and problems of superinfection [8].

European guidelines (and also those provided by ALAT) define high-risk patients as those who present with HAP/VAP and septic shock and/or the following risk factors for MDR pathogens: 1) admitted into units with high rates of MDR pathogens (i.e. above 25\%, including Gram-negative bacteria and MRSA); 2) prior use of antibiotics; 3 ) recent prolonged hospitalisation ( $>5$ days); and 4 ) previous colonisation by MDR pathogens [2].

The 2016 American guidelines indicate that when HAP is suspected, coverage for S. aureus, P. aeruginosa and other Gram-negative bacilli should be included in the empirical treatment [13]. The European guidelines indicate that, in certain patients, if a single antibiotic is effective against more than $90 \%$ of Gram-negative bacteria, the initial empirical therapy can be monotherapy if the patient is not in septic shock (table 2) [2].

Some studies suggest that survival in patients admitted to the ICU depends on the early initiation of effective antibiotic treatment $[2,14]$, while others indicate that combined treatments in critical patients 


\section{TABLE 2 Empirical antibiotic treatments for high-risk patients}

High risk of multidrug resistance and/ or $>15 \%$ risk of mortality in ICU
No septic shock

Septic shock
Monotherapy against Gram-negative (if active against $>90 \%$ of the probable ICU pathogens) \pm MRSA treatment

Dual treatment with Pseudomonas Gram-negative coverage \pm MRSA treatment

ICU: intensive care unit; MRSA: methicillin-resistant Staphylococcus aureus.

may be associated with increased toxicity and an increased risk of late-onset multidrug resistance and superinfection [2, 15-17].

When HAP/VAP is associated with septic shock or is produced by Gram-negative bacteria, the combination of two Gram-negative specific antibiotics may be beneficial $[18,19]$. Some studies even indicate that dual therapy ( $\beta$-lactams in combination with aminoglycosides, fluoroquinolones or macrolides/clindamycin) presents a significantly lower risk of death compared with monotherapy $[2,19]$.

In patients with a low risk of MDR and low mortality risk, the recommended therapy consists of a narrow-spectrum drug with activity against non-antibiotic-resistant Gram-negatives and MRSA. The appropriate drugs are ertapenem, cetriaxone, cefotaxime, moxifloxacin or levofloxacin $[2,13]$.

The percentage of MDR microorganisms isolated in each ICU should also be taken into account. According to European guidelines, in high-risk patients in an ICU in which $>25 \%$ of S. aureus respiratory isolates are methicillin-resistant (between $10 \%$ and $20 \%$ according to American guidelines) an agent with coverage for this pathogen should be added to the empirical treatment $[2,13]$.

Initially, a dual regimen should be used for P. aeruginosa in high-risk patients who are critically ill or with septic shock $[2,13]$. If the pathogen in the ICU may be Actinobacter spp., colistin should be used as the second agent. The agents for high-risk patients are displayed in table 3.

Key points

- It is important to start combined empirical treatment if there is a high risk of HAP/VAP due to MDR Gram-negative bacilli, and the patient has septic shock. If there is a high risk of MRSA in the unit, a specific antibiotic directed at this pathogen should be added [2].

- If combined therapy is started, it should be continued in the presence of pan-resistant or MDR non-fermenting Gram-negative bacteria or carbapenem-resistant Enterobacteria isolates [2].

- Combined therapy may be changed to monotherapy depending on the results of the cultures [2].

\section{Duration of treatment}

The duration of antibiotic treatment in HAP is a controversial point that has triggered spirited debate [5].

Several studies have compared different durations of antibiotic treatments. One study compared 8-day versus 15 -day treatment regimens, and found no differences in mortality (18.8\% in the group treated for 8 days and $17.2 \%$ in the group treated for 15 days). However, differences were observed in relapse of pneumonia. In particular, patients who had pneumonia due to non-fermenting Gram-negative bacilli, including Pseudomonas spp., in the 8-day regimen group presented more recurrences $(40.6 \%)$ than those

\section{TABLE 3 Agents for high-risk patients}

\begin{tabular}{lcc} 
Anti-Pseudomonal $\boldsymbol{\beta}$-lactams & Aminoglycosides & Anti-ESBL producers \\
\hline $\begin{array}{l}\text { Imipenem } \\
\text { Meropenem }\end{array}$ & $\begin{array}{c}\text { Amikacin (first line) } \\
\text { Gentamicin }\end{array}$ & Carbapenem (first line) \\
Cefepime & Tobramycin & Cefepime \\
Piperacillin/Tazobactam & & Piperacillin/Tazobactam \\
Ceftazidime & & Ceftazidime-Avibactam \\
Ceftolozane-Tazobactam & \\
\end{tabular}

ESBL: extended spectrum $\beta$-lactamase. \# : these two antibiotics were not commercialised during the guideline revision period and were not mentioned in the guidelines. 
treated with the 15-day regimen (25.4\%), although they presented less MDR pathogens [20]. Similarly, a Cochrane review assessing the efficacy of short versus long regimens (7-8 days versus 10-15 days) in patients with VAP concluded short-course antibiotic therapy does not increase the risk of adverse outcomes by non-fermenting Gram-negative bacilli; in addition, compared with longer treatments they may reduce MDR [21].

No differences were observed between the short and long treatments regarding the duration of mechanical ventilation or ICU stay [2].

Currently, both European and American guidelines recommend 7-8 day regimens in patients with VAP who do not have immunodeficiency, cystic fibrosis or other pulmonary problems such as pulmonary empyema, lung abscess, cavitation or necrotising pneumonia, and in those who respond well to antibiotic treatments [2].

A study using a 3-day course of antibiotics in patients with suspected HAP, but with low scores on the Clinical Pulmonary Infection Scale (CPIS) observed that this short course was associated with a reduced risk of superinfection or resistance compared with the standard duration of antibiotic treatment [21].

Rates of treatment discontinuation, due to adverse effects, appear to be similar in the two therapeutic options, although shorter treatments are likely to be associated with better tolerability [2].

Key points

- The current recommendation is to use 7-8 day treatments in patients with VAP without other respiratory comorbidities such as pulmonary empyema, lung abscess, cavitation or necrotising pneumonia, and who present a good response to treatment [2], in order to avoid the appearance of multidrug resistance. There are specific instances where longer durations of therapy may have benefit, as discussed later in this article (table 4).

- The European guidelines argue against routine antibiotic therapy for more than 3 days in patients with a low probability of HAP and no clinical deterioration within $72 \mathrm{~h}$ of symptom onset [2].

\section{Clinical assessment or detection of biomarkers as a predictor of response}

The ideal biomarker for appraising response to treatment is yet to be found. Theoretically, such a biomarker should be inexpensive, easily acquired and should provide results in a timely manner. This potential biomarker could help reduce antibiotic consumption and promote clinical research [1].

Several biomarkers for nosocomial pneumonia have been determined, including C-reactive protein, procalcitonin (PCT), copeptin and the mid-regional pro-atrial natriuretic peptide $[1,2]$. However, there are no clinical studies comparing treatment outcomes in HAP/VAP patients managed according to the patient's bedside clinical assessment or biomarker determinations [2].

In addition to the clinical signs of respiratory infection, the clinical assessment of the patient includes radiological tests, cultures of respiratory samples, analytical determinations and the scores from various rating scales such as the CPIS [2]. It is also known that the clinical criteria for the diagnosis of VAP have high sensitivity but low specificity; other complications in critically ill ventilated patients such as atelectasis, pulmonary oedema, pulmonary embolism and pulmonary trauma may closely resemble VAP [1].

The CPIS is a semi-objective evaluation of several clinical factors of pneumonia. A study assessing its ability to identify patients with VAP who might respond to treatment concluded that serial CPIS measurements might define the clinical course of VAP, identifying patients with a good response after 3 days from the diagnosis [22]. Another score used for the assessment of these patients is the Sequential Organ Failure Assessment (SOFA) score. A direct relationship has been observed between a decrease in the SOFA score and survival [23]. The same study concluded that the measurement of PCT and C-reactive protein at the beginning of VAP infection and on day 4 could predict mortality, and that a decrease in

TABLE 4 Patients in whom short antibiotic treatment is not possible and in whom treatment

should be individualised, and in whom procalcitonin may be useful to assess the efficacy and

duration of antibiotic treatment

Inadequate initial antibiotic treatment

Severely immunocompromised patients (neutropenia or stem cell transplant)

Highly antibiotic-resistant pathogens

Pseudomonas aeruginosa

Carbapenem-resistant Acinetobacter spp.

Carbapenem-resistant Enterobacteriaceae

Second-line antibiotic treatment (e.g. colistin, tigecycline) 
either one of these markers was a predictor of survival [23]. Similar results were observed in other studies which measured these markers on days 1, 3 and 7, confirming that PCT levels on day 3 were a strong predictor of mortality [2]. However, it should be emphasised that PCT has limited value in cases of renal failure, haemodialysis and resuscitated cardiac arrest [24].

For copetin, studies indicate that its levels at the onset of the VAP episode were lower in eventual survivors than in eventual non-survivors [2].

Key points

- There are no studies comparing management of HAP/VAP patients through sequential evaluation of biomarkers. The current recommendation is to use clinical criteria to decide whether or not to initiate antibiotic treatment or follow-up $[2,13]$.

- PCT alone is the greatest predictor of mortality risks [25].

- Biomarkers such as copeptin and the SOFA score can predict mortality in patients with VAP [25].

\section{PCT as a marker of treatment duration in patients with nosocomial pneumonia with} severe sepsis or VAP

Although PCT may be limited as a marker of treatment response, it has been abundantly studied and it is the most common biomarker evaluated in daily clinical practice [1]. Among the studies evaluating its usefulness for predicting VAP, one systematic review of seven studies found that high plasma PCT levels were associated with an increased risk of pneumonia [26]. Another study found that high baseline PCT levels were associated with lower survival [1].

A meta-analysis and a Cochrane review also indicated that the use of clinical algorithms based on PCT levels produce a significant reduction in antibiotic consumption without increasing mortality rates or treatment failure $[27,28]$

A later study conducted in China with 115 critically ill patients with VAP found that high PCT levels were associated with an increased risk of mortality within 2 months of diagnosis [29]. An open prospective study conducted with more than 1500 patients randomised to be treated following PCT results or with standard management, found that the average duration of antibiotic treatment in the PCT group was 5 days (range 3-9 days), compared with 4 days (range 4-11 days) in the standard antibiotic treatment group; 28 -day mortality rates were $20 \%$ and $25 \%$, respectively [30]. Other studies have shown that routine determination of serum PCT shortens the duration of antibiotic treatment by $\sim 3$ days and significantly lowers 28-day mortality [2].

It should be highlighted that inappropriate initial antibiotic treatment may delay the clinical response. Serial PCT measurement may help to plan the duration of antibiotic treatment, although this practice has not yet been validated for decision making upon initiation of treatment [2]. Similarly, it has not been possible to determine the shortest duration of appropriate antibiotic treatment in severely immunocompromised patients [2].

The European guidelines indicate that serial measurement of PCT serum levels can be performed together with clinical assessment in specific clinical circumstances (table 4) in order to shorten the duration of antibiotic treatment [2].

\section{Key points}

- Since the expected duration of antibiotic treatment in patients with HAP/VAP is 7-8 days, performing serial determinations of PCT levels has marginal effects on shortening antibiotic treatment in patients with a good response to initial treatment [2].

- Neither the initiation nor the duration of antibiotic treatment should depend solely on biomarkers [31].

\section{Topical application of non-absorbable antimicrobials in patients undergoing mechanical ventilation for more than $48 \mathrm{~h}$}

The oral flora of patients admitted to the hospital rapidly shifts to a predominance of aerobic Gram-negative pathogens that can be aspirated into the lower respiratory tract, ultimately resulting in the development of pneumonia. Therefore, appropriate hygiene of the oral cavity of hospitalised patients is mandatory. However, whether the use of oral antiseptics can reduce the incidence of VAP is still elusive [32].

Several systematic reviews have compared the use of oral chlorhexidine to prevent VAP in patients admitted to the ICU [2]. A meta-analysis of clinical trials including more than 3500 patients reported fewer respiratory infections in patients undergoing cardiac surgery (three studies) treated with oral chlorhexidine, although no differences in VAP were detected in studies of patients admitted to other ICUs [33]. Of note, oral chlorhexidine did not improve survival, duration of mechanical ventilation, or 
ICU length of stay in any ICU population (13 studies) [33]. In a later retrospective study of more than 5500 patients undergoing mechanical ventilation, the same authors reported that the daily use of chlorhexidine was associated with a lower risk of VAP, but higher mortality risk, possibly due to the aspiration of chlorhexidine and the development of acute respiratory distress syndrome [34].

Given these contradictory results, and the absence of a clear clinical benefit to offset the possible increase in mortality associated with chlorhexidine, the European guidelines did not issue a recommendation regarding its use [2].

To reduce the incidence of infectious complications in patients undergoing mechanical ventilation, selective oral or digestive decontamination (SOD and SDD, respectively) can be applied [2]. SDD involves the prevention of colonisation by both Gram-negative and Gram-positive bacteria and yeasts, through topical use of non-absorbable antibiotics in the oropharynx and the gastrointestinal tract, with or without parenteral antibiotic [3]. Oral decontamination with chlorhexidine is an alternative antiseptic in ecological niches with high levels of antibiotic resistance [3].

Studies comparing SOD with non-absorbable topical antibiotics indicate a reduction in the incidence of VAP, with no differences in the duration of mechanical ventilation or ICU stay. However, some studies found differences in mortality and others did not, sustaining controversy [2]. The effectiveness of SDD with non-absorbable topical antibiotics in the oropharynx and in the digestive tract has been evaluated in different clinical studies with a consistent reduction in mortality $[35,36]$.

Another meta-analysis evaluating resistance with the use of SOD and SDD or usual care indicated that there were no differences in the prevalence of colonisation or infection with resistant Gram-positives, including MRSA and vancomycin-resistant Enterococci, although a reduction of Gram-negative bacilli resistant to polymyxin and resistant to third-generation cephalosporins was found in patients who received selective decontamination [37].

In settings with low levels of antibiotic resistance, SOD (with non-absorbable topical antibiotics) and SDD (with administration of non-absorbable topical antibiotics in the oropharynx and gastrointestinal tract, and intravenous antibiotics) may be associated with reductions in nosocomial pneumonia and death. The potential effects of these antibiotics on antimicrobial resistance are uncertain [2].

\section{Key points}

- Because of the contradictory results reported, the European guidelines do not make any recommendations on the use of chlorhexidine for performing SOD in patients requiring mechanical ventilation [2].

- There are no major differences between benefits associated with SOD and SDD. Therefore, the European guidelines recommend the use of SOD rather than SDD, especially in environments with low resistance rates and low antibiotic consumption, in order to avoid the use of supplementary intravenous antibiotics [2].

Conflict of interest: A. Torres reports grants from MedImmune, Cubist, Bayer, Theravance, and from Poliphor; personal fees from Bayer (Advisory Board), Roche (Advisory Board), The Medicines CO (Advisory Board), and from Curetis (rapid molecular tests) (Advisory Board); grants as the Principal Investigator of a Phase III study of Cefatzidime/ Avibactam for HAP/VAP, during the conduct of the study. He has received personal fees from GSK (Educational symposium: Siglo XXI), Pfizer (speaker at sponsored vaccine symposia), Astra Zeneca (speaker at symposia on ceftaroline), and from Biotest Advisory Board (Enriched IgM for severe CAP), outside the submitted work. M.S. Niederman reports grants and personal fees from Bayer (inhaled amikacin research grant and consulting) and Merck (nosocomial pneumonia consulting and grant), personal fees from Thermo-Fisher (procalcitonin lectures), Theravance (consulting on telavancin), and Pfizer (consulting on linezolid), during the conduct of the study. J. Chastre reports personal fees from Medimmune/AstraZeneca (honoraria for consulting), Bayer (honoraria for consulting), Pfizer (honoraria for lecture), Cubist/Merck (honoraria for data safety committee participation), Aridis (honoraria for consulting and data safety participation), and Astellas (honoraria for consulting), outside the submitted work. G. Li Bassi reports grants from Medimmune, Cubist, Covidien, Theravance, Cardeas Pharma, Ciel Medical, Biovo Technologies, and Toray, during the conduct of the study. C. Luna reports grants and personal fees from Pfizer, and personal fees from Merck Sharp and Dohne, outside the submitted work. D. Rigau is a European Respiratory Society methodologist. T. Welte reports personal fees (for lectures/advisory board) from AstraZeneca, Basilea, Bayer, Grifols, Infectopharm, Novartis and Pfizer, research grants from Bayer, Grifols, Novartis and Pfizer, research grants from the EU, German Research Council and German Ministry of Research and Education, during the conduct of the study. He has received personal fees (for lectures/advisory board) from Boehringer, Berlin Chemie, Mundipharma and Insmed, outside the submitted work. R. Wunderink reports personal fees from Arsanis, Accelerate Diagnostics, Bayer, Cepheid, GenMark, The Medicines Company, Merck, Nabriva, Polyphor and Shionogi, during the conduct of the study. M. Kollef reports personal fees from Accelerate (consultant), Merck (consultant, speaker's bureau), Paratek (consultant) and Allergan (speaker's bureau), outside the submitted work. J.F. Timsit reports research grants from Merck and 3M, a research grant to his hospital from Astelas, and educational grants from Gilead; and personal fees (for advisory boards) from Pfizer, Paratek, Merck, Bayer, 3M and Abbott, all outside the submitted work. 


\section{References}

1 Salluh JIF, Souza-Dantas VC, Póvoa P. The current status of biomarkers for the diagnosis of nosocomial pneumonias. Curr Opin Crit Care 2017; 23: 391-397.

2 Torres A, Niederman MS, Chastre J, et al. International ERS/ESICM/ESCMID/ALAT guidelines for the management of hospital-acquired pneumonia and ventilator-associated pneumonia: Guidelines for the management of hospital-acquired pneumonia (HAP)/ventilator-associated pneumonia (VAP) of the European Respiratory Society (ERS), European Society of Intensive Care Medicine (ESICM), European Society of Clinical Microbiology and Infectious Diseases (ESCMID) and Asociación Latinoamericana del Tórax (ALAT). Eur Respir J 2017; 50: 1700582

3 Blanquer J, Aspa J, Anzueto A, et al. Normativa SEPAR: neumonía nosocomial [SEPAR guidelines for nosocomial pneumonia]. Arch Bronconeumol 2011; 47: 510-520.

4 Berton DC, Kalil AC, Teixeira PJZ. Quantitative versus qualitative cultures of respiratory secretions for clinical outcomes in patients with ventilator-associated pneumonia. Cochrane Database Syst Rev 2014; 10: CD006482.

5 Díaz E, Martín-Loeches I, Vallés J. Neumonía nosocomial [Nosocomial pneumonia]. Enferm Infecc Microbiol Clin 2013; 31: 692-698

6 Timsit JF, Harbarth S, Carlet J. De-escalation as a potential way of reducing antibiotic use and antimicrobial resistance in ICU. Intensive Care Med 2014; 40: 1580-1582.

7 Raman K, Nailor MD, Nicolau DP, et al. Early antibiotic discontinuation in patients with clinically suspected ventilator-associated pneumonia and negative quantitative bronchoscopy cultures. Crit Care Med 2013; 41: 1656-1663.

8 López-Pueyo MJ, Barcenilla-Gaite F, Amaya-Villar R, et al. Multirresistencia antibiótica en unidades de críticos [Antibiotic multiresistance in critical care units]. Med Intensiva 2011; 35: 41-53.

9 Martin-Loeches I, Torres A, Rinaudo M, et al. Resistance patterns and outcomes in intensive care unit (ICU)-acquired pneumonia. Validation of European Centre for Disease Prevention and Control (ECDC) and the Centers for Disease Control and Prevention (CDC) classification of multidrug resistant organisms. J Infect 2015; 74: $213-222$.

10 Verhamme KM, De Coster W, De Roo L, et al. Pathogens in early-onset and late-onset intensive care unit-acquired pneumonia. Infect Control Hosp Epidemiol 2007; 28: 389-397.

11 Arvanitis M, Anagnostou T, Kourkoumpetis TK, et al. The impact of antimicrobial resistance and aging in VAP outcomes: experience from a large tertiary care center. PLoS One 2014; 9: e89984.

12 Prina E, Ranzani OT, Polverino E, et al. Risk factors associated with potentially antibiotic-resistant pathogens in community-acquired pneumonia. Ann Am Thorac Soc 2015; 12: 153-160.

13 Kalil AC, Metersky ML, Klompas M, et al. Management of adults with hospital-acquired and ventilator-associated pneumonia: 2016 clinical practice guidelines by the Infectious Diseases Society of America and the American Thoracic Society. Clin Infect Dis 2016; 63: e61-e111.

14 Kumar A, Ellis P, Arabi Y, et al. Initiation of inappropriate antimicrobial therapy results in a fivefold reduction of survival in human septic shock. Chest 2009; 136: 1237-1248.

15 Kett DH, Cano E, Quartin AA, et al. Implementation of guidelines for management of possible multidrug-resistant pneumonia in intensive care: an observational, multicentre cohort study. Lancet Infect Dis 2011; 11: 181-189.

16 Bliziotis IA, Samonis G, Vardakas KZ, et al. Effect of aminoglycoside and beta-lactam combination therapy versus beta-lactam monotherapy on the emergence of antimicrobial resistance: a meta-analysis of randomized, controlled trials. Clin Infect Dis 2005; 41: 149-158.

17 Koontz CS, Chang MC, Meredith JW. Effects of empiric antibiotic administration for suspected pneumonia on subsequent opportunistic pulmonary infections. Am Surg 2000; 66: 1110-1114.

18 Kumar A, Safdar N, Kethireddy S, et al. A survival benefit of combination antibiotic therapy for serious infections associated with sepsis and septic shock is contingent only on the risk of death: a meta-analytic/meta-regression study. Crit Care Med 2010; 38: 1651-1664.

19 Kumar A, Zarychanski R, Light B, et al. Early combination antibiotic therapy yields improved survival compared with monotherapy in septic shock: a propensity-matched analysis. Crit Care Med 2010; 38: 1773-1785.

20 Chastre J, Wolff M, Fagon JY, et al. Comparison of 8 ss 15 days of antibiotic therapy for ventilator associated pneumonia in adults. JAMA 2003; 290: 2588-2598.

21 Pugh R, Grant C, Cooke RP, et al. Short-course versus prolonged-course antibiotic therapy for hospital-acquired pneumonia in critically ill adults. Cochrane Database Syst Rev 2015; 8: CD007577.

22 Luna CM, Blanzaco D, Niederman MS, et al. Resolution of ventilator-associated pneumonia: prospective evaluation of the clinical pulmonary infection score as an early clinical predictor of outcome. Crit Care Med 2003; 31: 676-682.

23 Seligman R, Meisner M, Lisboa TC, et al. Decreases in procalcitonin and C-reactive protein are strong predictors of survival in ventilator-associated pneumonia. Crit Care 2006; 10: R125.

24 Amour J, Bienbaum A, Langeron O, et al. Influence of renal dysfunction on the accuracy of procalcitonin for the diagnosis of postoperative infection after vascular surgery. Crit Care Med 2008; 36: 1147-1154.

25 Seligman R, Seligman BGS, Teixeira PJ. Comparing the accuracy of predictors of mortality in ventilator-associated pneumonia. J Bras Pneumol 2011; 37: 495-503.

26 Sotillo-Díaz JC, Bermejo-López E, García-Olivares P, et al. Papel de la procalcitonina plasmatica en el diagnostico de la neumonia asociada a ventilacion mecanica: revision sistematica y metaanalisis [Role of plasma procalcitonin in the diagnosis of ventilator-associated pneumonia: systematic review and metaanalysis]. Med Intensiva 2014; 38: 337-346.

27 Schuetz P, Chiappa V, Briel M, et al. Procalcitonin algorithms for antibiotic therapy decisions. Arch Intern Med 2011; 171: 1322-1331.

28 Schuetz P, Müller B, Stolz D, et al. Procalcitonin to initiate or discontinue antibiotics in acute respiratory tract infections. Cochrane Database Syst Rev 2012; 9: CD007498.

29 Li B, Zhao X, Li S. Serum procalcitonin level and mortality risk in critically ill patients with ventilator-associated pneumonia. Cell Physiol Biochem 2015; 37: 1967-1972.

30 de Jong E, van Oers JA, Beishuizen A, et al. Efficacy and safety of procalcitonin guidance in reducing the duration of antibiotic treatment in critically ill patients: a randomised, controlled, open-label trial. Lancet Infect Dis 2016; 16: 819-827. 
31 Nora D, Salluh J, Martin-Loeches I, et al. Biomarker-guided antibiotic therapy-strengths and limitations. Ann Transl Med 2017; 5: 208.

32 Vergara Messina TM. Descontaminación oral en la prevención de neumonía asociada a ventilación mecánica [Oral decontamination in the prevention of ventilator-associated pneumonia]. Medwave 2010; 10: e4621.

33 Klompas M, Speck K, Howell MD, et al. Reappraisal of routine oral care with chlorhexidine gluconate for patients receiving mechanical ventilation: systematic review and meta-analysis. JAMA Intern Med 2014; 174: 751-761.

34 Klompas M, Li L, Kleinman K, et al. Associations between ventilator bundle components and outcomes. JAMA Intern Med 2016; 176: 1277-1283.

35 Stolz D, Smyrnios N, Eggimann P, et al. Procalcitonin for reduced antibiotic exposure in ventilator-associated pneumonia: a randomised study. Eur Respir J 2009; 34: 1364-1367.

36 D'Amico R, Pifferi S, Torri V, et al. Antibiotic prophylaxis to reduce respiratory tract infections and mortality in adults receiving intensive care. Cochrane Database Syst Rev 2009; 4: CD000022.

37 Daneman N, Sarwar S, Fowler RA, et al. Effect of selective decontamination on antimicrobial resistance in intensive care units: a systematic review and meta-analysis. Lancet Infect Dis 2013; 13: 328-341. 